SAND96-0350 • UC-706

Unlimited Release

Printed August 1996

\title{
Time-Dependent Electron Temperature Diagnostics for High-Power Aluminum z-Pinch Plasmas
}

T. W. L. Sanford, K. G. Whitney, T. J. Nash, R. C. Mock, J. P. Apruzese, T. L. Gilliland, D. O. Jobe, J. S. McGurn, P. E. Pulsifer, J. F. Seamen, R. B. Spielman, J. W. Thornhill, M. F. Vargas

\author{
Prepared by \\ Sandia National Laboratories \\ Albuquerque, New Mexico 87185 and Livermore, California 94550 \\ for the United States Department of Energy \\ under Contract DE-AC04-94AL85000
}

Approved for public release; distribution is unlimited. 
Issued by Sandia National Laboratories, operated for the United States Department of Energy by Sandia Corporation.

NOTICE: This report was prepared as an account of work sponsored by an agency of the United States Government. Neither the United States Government nor any agency thereof, nor any of their employees, nor any of their contractors, subcontractors, or their employees, makes any warranty, express or implied, or assumes any legal liability or responsibility for the accuracy, completeness, or usefulness of any information, apparatus, product, or process disclosed, or represents that its use would not infringe privately owned rights. Reference herein to any specific commercial product, process, or service by trade name, trademark, manufacturer, or otherwise, does not necessarily constitute or imply its endorsement, recommendation, or favoring by the United States Government, any agency thereof or any of their contractors or subcontractors. The views and opinions expressed herein do not necessarily state or reflect those of the United States Government, any agency thereof or any of their contractors.

Printed in the United States of America. This report has been reproduced directly from the best available copy.

Available to DOE and DOE contractors from

Office of Scientific and Technical Information

PO Box 62

Oak Ridge, TN 37831

Prices available from (615) 576-8401, FTS 626-8401

Available to the public from

National Technical Information Service

US Department of Commerce

5285 Port Royal Rd

Springfield, VA 22161

NTIS price codes

Printed copy: $\mathrm{A} 03$

Microfiche copy: A01 


\title{
Time-Dependent Electron Temperature Diagnostics for High-Power Aluminum z-Pinch Plasmas*
}

\author{
T. W. L. Sanford, T. J. Nash, R. C. Mock, R. B. Spielman, J. F. Seamen,
} J. S. McGurn, D. O. Jobe, T. L. Gilliland, and M. F. Vargas

Sandia National Laboratories, Albuquerque, NM 87185

K. G. Whitney, J. W. Thornhill, P. E. Pulsifer, and J. P. Apruzese

Naval Research Laboratory, Radiation Hydrodynamics Branch, Washington, DC 20375 USA

\section{Abstract}

Time-resolved $\mathrm{x}$-ray pinhole photographs and time-integrated radially-resolved $\mathrm{x}$-ray crystal-spectrometer measurements of azimuthally-symmetric aluminum-wire implosions suggest that the densest phase of the pinch is composed of a hot plasma core surrounded by a cooler plasma halo. The slope of the free-bound $\mathrm{x}$-ray continuum, provides a time-resolved, model-independent diagnostic of the core electron temperature. A simultaneous measurement of the time-resolved $\mathrm{K}$-shell line spectra provides the electron temperature of the spatially averaged plasma. Together, the two diagnostics support a 1-D Radiation-Hydrodynamic model prediction of a plasma whose thermalization on axis produces steep radial gradients in temperature, from temperatures in excess of a kilovolt in the core to below a kilovolt in the surrounding plasma halo.

*Presented at the 11th APS Topical Conference on High-Temperature Plasma Diagnostics (Monterey, CA, May 13-16, 1996). 


\section{Acknowledgments}

We thank J. E. Maenchen, M. K. Matzen, D. H. McDaniel, and J. Davis (NRL) for vigorous programmatic support; G. A. Allshouse for reviewing and L. O. Peterson for typing this manuscript. 


\section{DISCLAIMER}

Portions of this document may be illegible in electronic image products. Images are produced from the best available original document. 


\section{Contents}

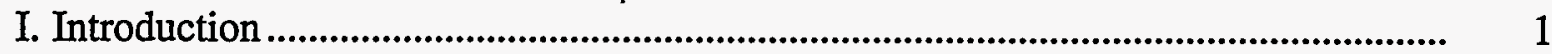

II. Experimental Arrangement ........................................................................................ 1

III. Global Implosion Characteristics ........................................................................... 2

IV. Core-Temperature Diagnostic .................................................................................... 4

V. Halo-Temperature Diagnostic and Model ................................................................. 7

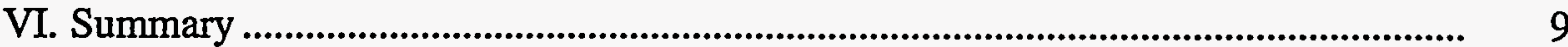

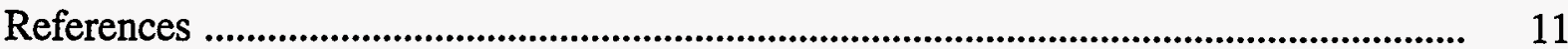

\section{Figures}

1A. Radial profile measured in the $\mathrm{PHC}$ near peak compression showing the plasma core and halo spatial distribution.

1B. Relative comparison of the radial profile of the free-bound continuum between 4 and $5.4 \AA$ with the radial profile of the hydrogen alpha line, as measured in the radially-resolved crystal spectrometer (see also Fig. 3).

2A. Relative comparison of the radiation measured in $\mathrm{XRD}(0.25 \mathrm{keV}), \mathrm{PCD}(2.5 \mathrm{keV})$, and $\operatorname{PCD}(6.2 \mathrm{keV})$ for one of the 90 -wire shots, showing that the second pulse has a lower temperature than the first. See Table I for detector definitions.

2B. Comparison of the FWHM of the core and halo (as defined in Fig. 1A) for the three 90-wire shots measured in the PCD, showing a strong and weak compression of the core, which merges with the halo after the first and second compression. The halo shown as the dashed lines between 0 and 24 ns corresponds to the mean and RMS variation of the halo measured over that period for the three shots.

2C. The electron temperature of the core extracted from the filtered PCD measurements for the 90-wire shot shown in Fig. 2A. The small percentage error-bar corresponds to the typical $\pm 12 \%$ error of the fit. The large percentage error-bar corresponds to the systematic $23 \%$ uncertainty in extracting the temperature associated with the limited number of PCDs used.

2D. Measured line ratios of the hydrogen-like Lyman-alpha to helium-like $n=2$ to $n=1$ resonance lines are shown for each of the three 90-wire shots as a function of time, together with the radiation measured from $\operatorname{PCD}(2.5 \mathrm{keV})$ for one of the 90 -wire shots.

2E. The electron temperatures that are inferred from the data in Figs. 2A corresponding to $\mathrm{PCD}(2.5 \mathrm{keV})$, Fig. 2B corresponding to core FWHM, and Fig. 2D are shown for each of the three 90 -wire shots.

2F. The ion densities that are inferred from the data in Figs. 2A (corresponding to PCD $(2.5 \mathrm{keV})$ ), Fig. 2B (corresponding to the core FWHM), and Fig. 2D are shown for each of the three 90-wire shots.

3. Comparison of the on-axis and 3-mm off-axis spectra measured side on in the radially-resolved crystal spectrometer. 
4. The slope of the free-bound continuum as measured in the filtered PCDs for the 136 -wire shot 2239 . The horizontal error bars correspond to the $\pm 25 \%$ energy response band about the given average-energy response and the vertical error bars correspond-to the $\pm 13 \%$ relative PCD calibration uncertainty.

5. Relative response of the XRD and PCDs shown in Fig. 2A and used in extracting the core electron temperature (see also Table I). 


\section{Time-Dependent Electron Temperature Diagnostics for High-Power Aluminum z-Pinch Plasmas}

\section{Introduction}

Knowledge of the time-dependent electron temperature in $\mathrm{z}$ pinches is required to understand pinch dynamics and radiation generation. In this paper, two timedependent electron-temperature diagnostics are discussed and applied to $~ 30-T W$ aluminum z-pinch plasmas generated by imploding large-wire-number arrays ${ }^{1}$ on the Saturn accelerator. ${ }^{2}$ The use of large wire numbers reduces the azimuthal asymmetry of the initial plasma, improves shot-to-shot repeatability, and permits, for the first time, details of wire implosions to be meaningfully compared with $2 \mathrm{D}$-numerical simulations over the full span of the $\mathrm{x}$-ray emission. ${ }^{3}$

Our measurements indicate that these implosions are characterized by a hot plasma core that is surrounded by a cooler plasma halo. A similar model was proposed to explain temperature differences seen in $\mathrm{Mg} / \mathrm{Al}$ mixture experiments. ${ }^{4}$ In this paper, we show firstly that the measurement of the free-bound (FB) $\mathrm{x}$ rays from the highly-stripped, $\mathrm{K}$-shell aluminum ions provides a model-independent measurement of the core electron temperature owing to the long $\mathrm{x}$-ray mean-free-path and to the observed FB $\mathrm{x}$-ray origin in the core. Secondly, we show that the simultaneous measurement of the softer line spectra, which is sensitive to effects of plasma $x$-ray opacity, provides a spatially averaged measurement of the electron temperature. Before these two diagnostics are discussed, the overall experimental arrangement and global characteristics of the implosion are reviewed.

\section{Experimental Arrangement}

The experimental arrangement was identical to that of Ref. 1 and similar to that of Ref. 5, except for the number of wires in the array and diagnostics used. Here, the load consisted of an annular array of 90 or 136 aluminum wires mounted at a radius of $8.6 \mathrm{~mm}$, having a length of $20 \mathrm{~mm}$, and a total mass of $\sim 615 \mu \mathrm{g}$. The 90-wire experiments were repeated three times, in shots numbered 2085, 2094, and 2095, and found to be highly reproducible. Eight current-return posts located at a radius of $17.3 \mathrm{~mm}$ permitted the radiation from the load to be measured in detectors located at the ends of three vacuum linesof-sight positioned $55^{\circ}$ off the pinch axis. With this load, a peak current of $6.9 \pm 0.2 \mathrm{MA}$ with a 35-ns 10-to-90\% rise time was measured 45-mm upstream of the load. These detectors included a spatially-integrating time-resolved bolometer, filtered $\mathrm{x}$-ray diodes (XRDs) and photoconducting detectors (PCDs), a time-resolved x-ray pinhole camera (PHC) (with 1-ns and $150-\mu \mathrm{m}$ temporal and spatial resolution, respectively) filtered by $25-\mu \mathrm{m} \mathrm{Be}$, a time- 
resolved KAP-crystal spectrometer (with 1-ns and 3-eV temporal and energy resolution, respectively), and a radially-resolved time-integrating KAP-crystal spectrometer (with better than 200- $\mu \mathrm{m}$ and 10-eV spatial and energy resolution, respectively). The PHC was sensitive to $\mathrm{X}$ rays greater than $\sim 1 \mathrm{keV}$, and the bolometer, XRDs, PCDs, and time-resolved spectrometer spatially integrated the radiation generated from over $70 \%$ of the implosion.

\section{Global Implosion Characteristics}

In general, the radial emission profiles measured near stagnation seen by the PHC are well fit by the sum of two Gaussian distributions when integrated over the axial length of the pinch, as illustrated in Fig. 1A. This figure shows the profile measured within $1 \mathrm{~ns}$ of peak compression for one of the three 90 -wire shots measured. It illustrates the presence of an intense Gaussian-like core having a 0.62-mm FWHM (full-width half-maximum) surrounded by a Gaussian-like halo with a 3.2-mm FWHM. At peak compression, the associated axial variation is typically less than $\pm 10 \%$ over the $15-\mathrm{mm}$ pinch length measured, when integrated over radius. At later times an $m=0$ instability is observed to form, resulting in a $\pm 25 \%$ variation in axial intensity that has a $3 \pm 1-\mathrm{mm}$ period. This axial variation and its implications are ignored in the present analysis.

The radiation from one of the shots, together with the time evolution of the widths of the core and halo measured for the three shots, are shown in Fig. 2A and 2B, respectively. The data show that the strong and weak radiation pulses correlate well with the strong and weak pinches of the core. The radially-resolved time-integrated spectrometer indicates that the FB radiation originates predominantly from the core; whereas, the spectrometer shows that the source of the $\mathrm{K}$-line emission includes core plasma as well as plasma well into the halo region (Fig. $1 B$ and 3). Both the presence of the significant FB emission (with its slope indicating $\mathrm{keV}$ temperatures) at small radii, lack of significant FB emission at larger radii,
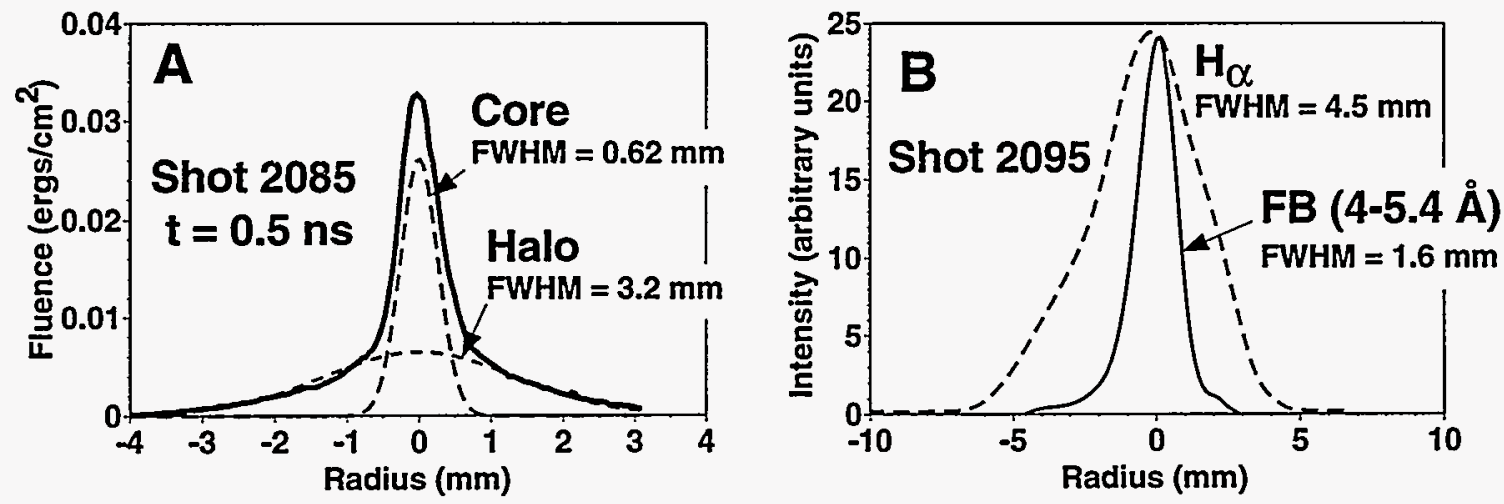

Figure 1A. Radial profile measured in the PHC near peak compression showing the plasma core and halo spatial distribution.

1B. Relative comparison of the radial profile of the free-bound continuum between 4 and $5.4 \AA$ with the radial profile of the hydrogen alpha line, as measured in the radially-resolved crystal spectrometer (see also Fig. 3). 

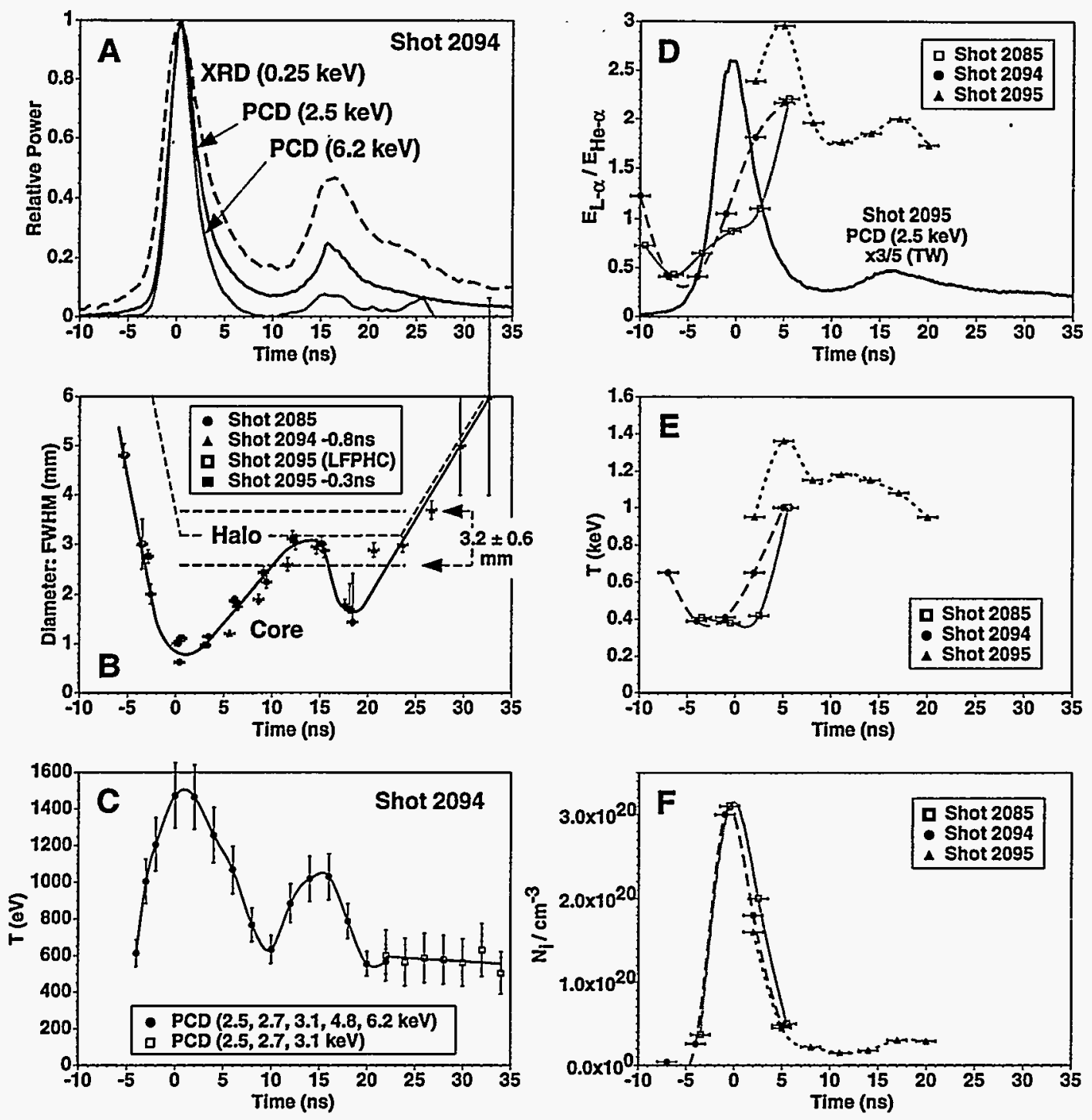

Figure 2A. Relative comparison of the radiation measured in $\mathrm{XRD}(0.25 \mathrm{keV}), \mathrm{PCD}(2.5 \mathrm{keV})$, and $\mathrm{PCD}(6.2$ $\mathrm{keV}$ ) for one of the 90-wire shots, showing that the second pulse has a lower temperature than the first. See Table I for detector definitions.

2B. Comparison of the FWHM of the core and halo (as defined in Fig. 1A) for the three 90-wire shots measured in the PCD, showing a strong and weak compression of the core, which merges with the halo after the first and second compression. The halo shown as the dashed lines between 0 and $24 \mathrm{~ns}$ corresponds to the mean and RMS variation of the halo measured over that period for the three shots.

2C. The electron temperature of the core extracted from the filtered PCD measurements for the 90-wire shot shown in Fig. 2A. The small percentage error-bar corresponds to the typical $\pm 12 \%$ error of the fit. The large percentage error-bar corresponds to the systematic $23 \%$ uncertainty in extracting the temperature associated with the limited number of PCDs used.

2D. Measured line ratios of the hydrogen-like Lyman-alpha to helium-like $n=2$ to $n=1$ resonance lines are shown for each of the three 90-wire shots as a function of time, together with the radiation measured from $\operatorname{PCD}(2.5 \mathrm{keV})$ for one of the 90 -wire shots.

2E. The electron temperatures that are inferred from the data in Figs. $2 \mathrm{~A}$ corresponding to $\mathrm{PCD}(2.5$ $\mathrm{keV}$ ), Fig. 2B corresponding to core FWHM, and Fig. 2D are shown for each of the three 90-wire shots.

2F. The ion densities that are inferred from the data in Figs. 2A (corresponding to $P C D(2.5 \mathrm{keV})$ ), Fig. 2B (corresponding to the core FWHM), and Fig. 2D are shown for each of the three 90-wire shots. 


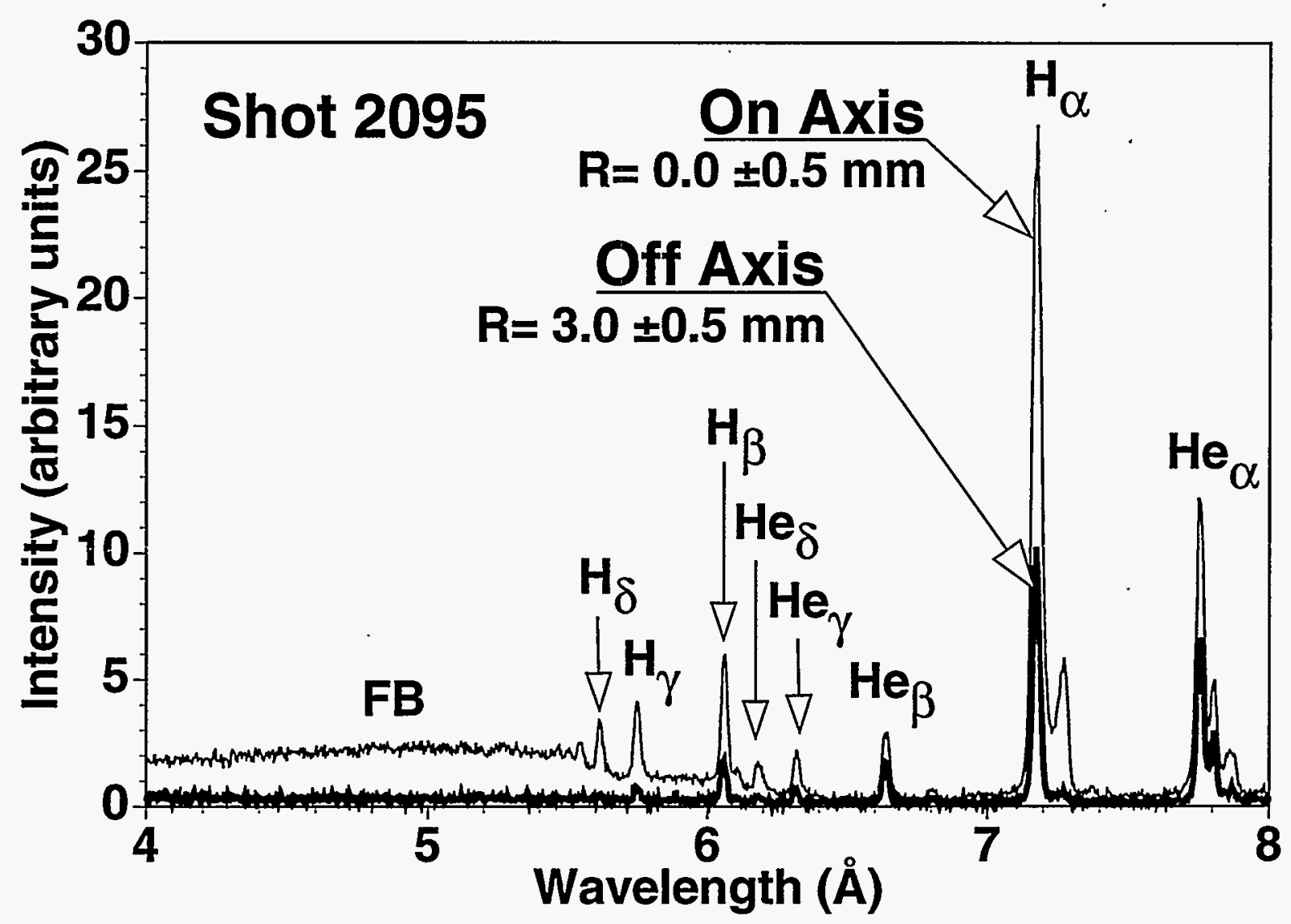

Figure 3. Comparison of the on-axis and 3-mm off-axis spectra measured side on in the radially-resolved crystal spectrometer.

and the decreasing $\mathrm{H} \alpha$ to $\mathrm{He} \alpha$ line-ratios with radius indicate that the core is hotter than the halo, on average. Additionally, because the measured $\mathrm{x}$-ray yield generated during the second compression relative to that generated during the first compression decreases as the energy of the $x$ ray increases (Fig. 2A), the data also imply that the core of the second compressions is cooler than that of the first, on average.

The next two sections support these observations. They describe how the PCD and the spectrometer diagnostics are used to determine and to infer the electron temperature of the core and halo, respectively.

\section{Core-Temperature Diagnostic}

For a Maxwellian plasma of electron temperature T, the energy spectrum of the freebound $(\mathrm{FB}) \mathrm{x}$ rays is proportional to $\exp (-\mathrm{E} / \mathrm{T})$, where $\mathrm{E}$ is the energy of the given $\mathrm{x}$ ray. Here, the slope of this FB spectrum and thus the associated electron temperature is measured with a set of filtered PCDs, ${ }^{6}$ as shown, for example, in Fig. 4 for a 136-wire shot number 
2239. For this shot, the data give an electron temperature of $1.3 \pm 0.2 \mathrm{keV}$ at peak power. The $\mathrm{x}$-ray energy sensitivity of the filtered PCDs used is calculated using XRDNEW, ${ }^{7}$ an $\mathrm{X}$-ray detector design code, and is shown in Fig. 5, with the mean energy and bandwidth response tabulated in Table I. Although the PCDs lack the ability to resolve the radiation spatially, and because the time-integrated crystal-spectrometer data show that the majority of the FB radiation originates at radii less than $1 \mathrm{~mm}$ (Figs. $1 \mathrm{~B}$ and 3), the temperature extracted is thus assumed to be associated with the core plasma. The presence of non-Maxwellian electrons and beam plasma interactions could potentially corrupt the interpretation of this PCD slope measurement.

\section{Table I}

Mean energy response $\bar{E}$ and associated band width $\pm \Delta E$ over which $\pm 25 \%$ of the $x$ rays are observed about $\overline{\mathrm{E}}$ in the filtered XRD shown in Fig. $2 \mathrm{~A}$ and the filtered PCDs used to extract the electron temperature of Fig. 4.

\begin{tabular}{|c|c|c|c|c|}
\hline Name & Filter & $\underset{(\mathbf{k e V})}{\overline{\mathrm{E}}}$ & $\begin{array}{c}+\Delta E \\
(\mathrm{keV})\end{array}$ & $\frac{-\Delta \mathbf{E}}{(\mathbf{k e V})}$ \\
\hline $\mathrm{XRD}(0.25 \mathrm{keV})$ & 1- $\mu \mathrm{m}$ Kimfol & $\sim 0.25$ & $\sim 0.35$ & $\sim 0.12$ \\
\hline $\operatorname{PCD}(2.5 \mathrm{keV})$ & $8-\mu \mathrm{m} \mathrm{Be}$ & 2.5 & 0.75 & 0.64 \\
\hline PCD $(2.7 \mathrm{keV})$ & 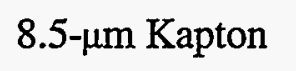 & 2.7 & 0.80 & 0.56 \\
\hline $\operatorname{PCD}(3.1 \mathrm{keV})$ & $25-\mu \mathrm{m}$ Kapton & 3.1 & 0.74 & 0.56 \\
\hline $\operatorname{PCD}(3.5 \mathrm{keV})$ & $50-\mu \mathrm{m}$ Kapton & 3.5 & 0.91 & 0.65 \\
\hline $\mathrm{PCD}(4.2 \mathrm{keV})$ & $127-\mu \mathrm{m}$ Kapton & 4.2 & 0.90 & 0.70 \\
\hline $\operatorname{PCD}(4.8 \mathrm{keV})$ & $254-\mu \mathrm{m}$ Kapton & 4.8 & 0.90 & 0.70 \\
\hline PCD $(6.2 \mathrm{keV})$ & $25-\mu \mathrm{m} \mathrm{Ge}$ & 6.2 & 1.00 & 0.80 \\
\hline
\end{tabular}

Before and after the measurements discussed in this paper, the relative sensitivity of each PCD diamond element was established to a precision of $\pm 13 \%$ by exposing each element to the same filtered $\mathrm{x}$-ray fluence from aluminum-wire shots. The absolute response of a given element is tied back (via this relative technique) to detectors calibrated at the Brookhaven National Syncrotron Light Source to a precision of 20\%. The time-integrated spectrum (determined from a KAP crystal spectrometer over the measured energy range 0.9 to $3.5 \mathrm{keV}$ ) and its extension to high x-ray energies when convoluted with the detector sensitivity (Fig. 5) is used to evaluate the energy response of the given detector shown in Table I. Between 3.0 and $3.5 \mathrm{keV}$, the slope of the FB spectra for typical aluminum wire shots is measured to be $\sim 1.3 \mathrm{keV}$, which is used to estimate the filtered PCD response above 3 $\mathrm{keV}$. Although each detector's response at a given time is dependent on the exact spectrum at that time, because the detector response varies simultaneously in a similar fashion for all the detectors used, the fitted slope (as shown in Fig. 4) is relatively insensitive to the exact spectrum used to estimate the ensemble of responses. The resulting uncertainty in temperature, caused by not iterating the detector response, is estimated to be substantially less than the $12 \%$ uncertainty of the fit itself and, thus, response iteration is ignored. 


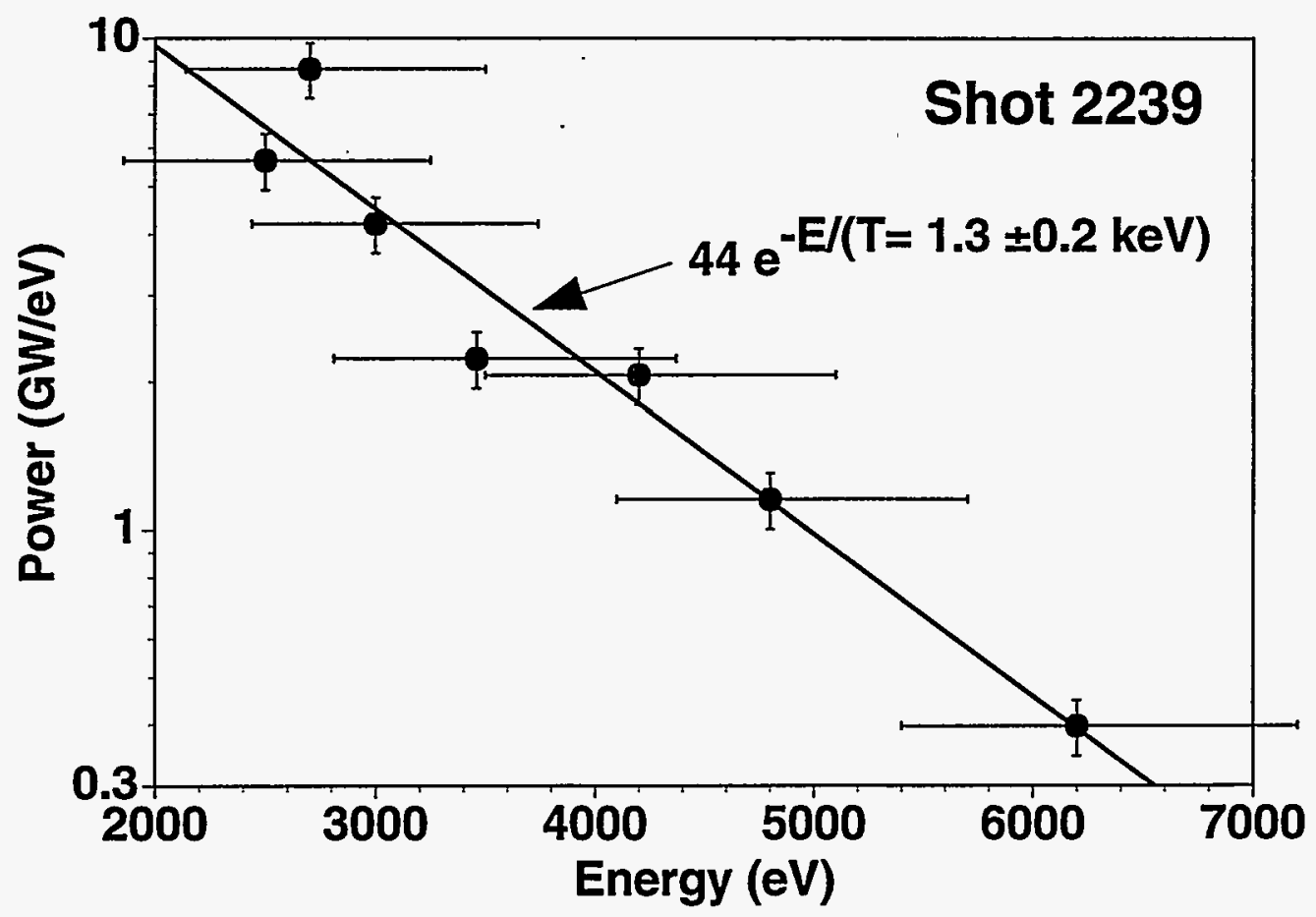

Figure 4. The slope of the free-bound continuum as measured in the filtered PCDs for the 136-wire shot 2239 . The horizontal error bars correspond to the $\pm 25 \%$ energy response band about the given average-energy response and the vertical error bars correspond to the $\pm 13 \%$ relative $P C D$ calibration uncertainty.

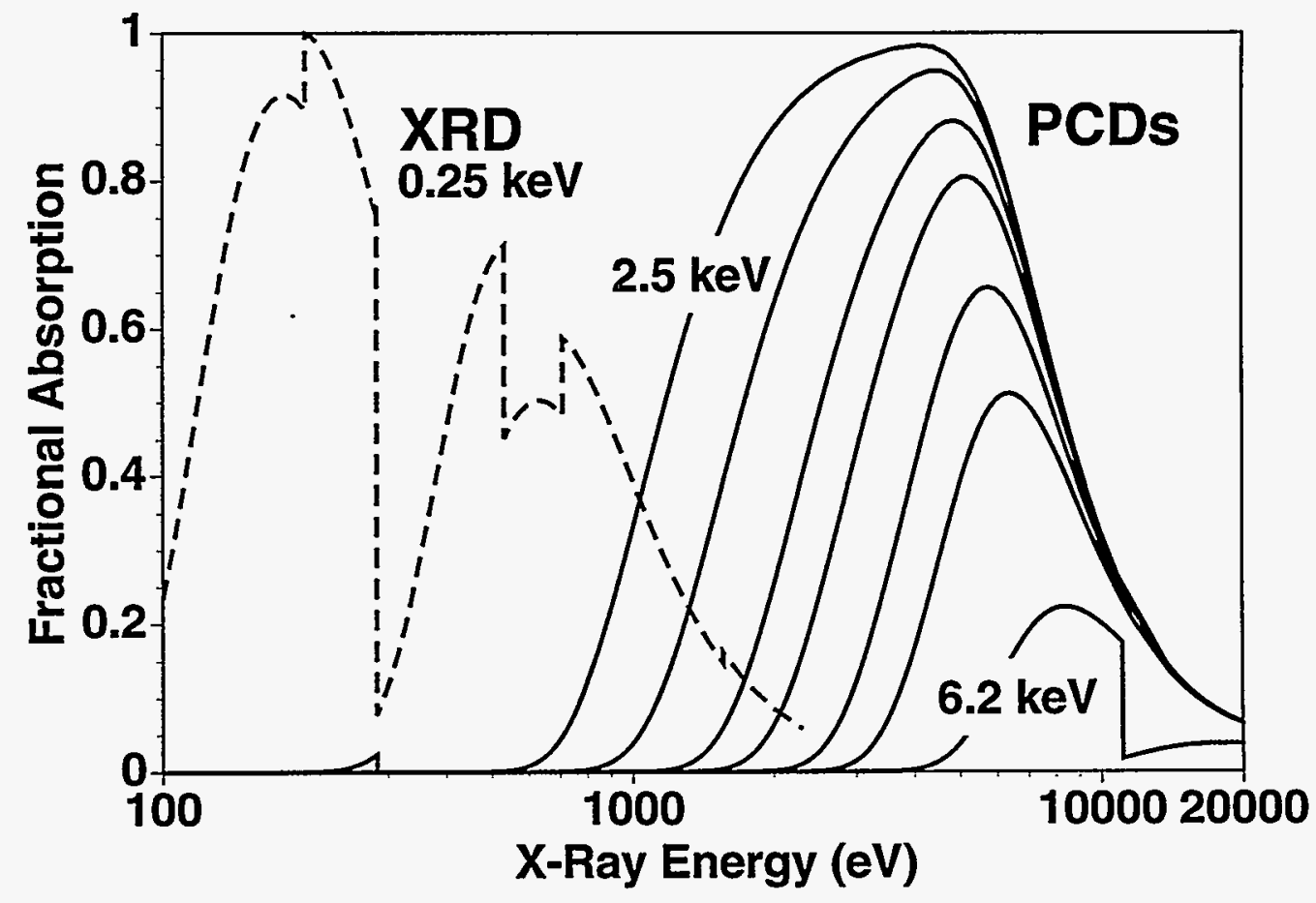

Figure 5. Relative response of the XRD and PCDs shown in Fig. 2A and used in extracting the core electron temperature (see also Table I). 
Using this technique, the temperature extracted from one of the 90 -wire shots as a function of time is shown in Fig. 2C. The two peak temperatures correlate well with the times of the two pinches and radiation peaks, as might be expected. For the 90 -wire shots, PCD (3.5 keV) and PCD (4.2 keV) were not available and accordingly were not used in extracting the temperatures shown. Additionally, in Fig. $2 \mathrm{C}$ beyond $24 \mathrm{~ns}$, the signals from the highly filtered PCDs become too weak to be measured above background, and thus only PCD (2.5 keV), PCD (2.7 keV), and PCD (3.1 keV) were fit, resulting in a $23 \%$ systematic uncertainty in extracting the temperature beyond $24 \mathrm{~ns}$. The temperature measured at peak compression for the three shots is $1.4 \pm 0.1 \mathrm{keV}$ in agreement with that measured for the 136wire shot. The uncertainty refers to the RMS shot-to-shot variation and reflects on the excellent reproducibility of the implosions.

\section{Halo-Temperature Diagnostic and Model}

The time-resolved pinhole pictures show a shell of plasma imploding to the axis (with an axial precursor), merging to form a cylinder of plasma. As shown in Figs. $1 \mathrm{~A}$ and $2 \mathrm{~B}$, the core of this cylinder pinches to a diameter of less than $1 \mathrm{~mm}$ and rebounds to a diameter of $3 \mathrm{~mm}$ before undergoing a secondary pinch to a diameter of $2 \mathrm{~mm}$. The pinhole pictures record the entire kilovolt spectrum of emitted $x$ rays, so they give no direct measure of the size of the temperature and density gradients that may be present in the plasma; however, they do give a quantitative measure of the size of the $\mathrm{K}$-shell emission region.

One can get a sense of the presence and magnitude of the temperature gradients, however, by using different spectral measurements to infer temperature. One-D simulations ${ }^{8}$ of the 90-wire implosions that produced the best agreement with the experimental data suggest that gradients in temperature are formed when a shell of plasma implodes on a precursor plasma, which sweeps up and compresses the precursor into the innermost region of a core that is then heated to much higher temperatures than the surrounding halo. The precursor is ionized in advance of the shell along with the resulting core to mostly fully stripped aluminum. Continuum emission is expected to originate from the hotter regions, which are being compressed by the cooler current sheath. Optically-thick lines, on the other hand, that are emitted from hot regions will be absorbed and re-emitted from cooler regions. Thus, when time-resolved line spectra taken from shots 2085, 2094, and 2095 are used to infer spatially-averaged temperatures, a comparison of these temperatures with those inferred from (the PCD derived) continuum-slope measurements should provide information on the temperature gradients present in the plasma.

The basic procedure we used for inferring both temperatures and densities as a function of time from time-resolved line emission data, similar to the time-integrated data of Fig. 3, is described in Ref. 9 and further evaluated in Ref. 10. This procedure has also been applied to z-pinch data analyses in Refs. 4 and 11. A unique determination of both quantities is possible if one requires that the calculated power output from $\mathrm{K}$-shell emissions as well as the calculated energy ratio of hydrogen-like Lyman-alpha to helium-like $n=2$ to $n=1$ emissions be simultaneously in agreement with the corresponding time-resolved measured values of these quantities. The calculations are time dependent and done with a minimum of 
assumptions about the spatial structure or temporal history of the plasma. Specifically, a uniform plasma is assumed, which is taken to be the size of the measured plasma, and the plasma is assumed to be in collisional-radiative equilibrium (CRE). The ionization equilibrium calculation self-consistently includes the opacity effects of photo-excitations, deexcitations, and ionizations.

The radial sizes used for this analysis correspond to those measured for the core in Fig. 2B and the K-shell powers used at each time are obtained from Fig. 2A corresponding to PCD $(2.5 \mathrm{keV})$. The use of the core radius is a first approximation, since we know that the lines are emitted from the halo as well. The Ly-alpha/(He-alpha+He-IC) line energy ratios obtained from the time-resolved spectra of the three 90-wire shots are shown in Fig. 2D together with the K-shell power pulse measured for one of the shots (similar to that of Fig. $2 \mathrm{~A}$ ). These data show that helium-like line emission dominates over hydrogen-like line emission up until peak compression, which coincides with the peak of the power pulse. After this time, as the plasma begins to expand, hydrogen-like emissions rise rapidly. The plasma either stays hot throughout the time interval of expansion and recontraction (in contradiction with the electron temperature measured from the continuum slope (Fig. 2C)), or it expands and cools too quickly for the totally ionized and hydrogen-like ionization stages to recombine significantly. That is, the data shows that the hydrogen-like lines continue to dominate the helium-like lines even as the core plasma expands, cools, recompresses, and heats. Because this line behavior implies recombination of the highly ionized states is slow in the core, the continuum emission into hydrogenic aluminum is concentrated in the highly ionized core of the plasma during peak compression. However, its slope reflects the electron temperature. Thus, because Fig. $2 \mathrm{C}$ shows that the electrons are cooling due to heat conduction, radiation losses, and expansion, it also implies that the ions and electrons in the core are not remaining in CRE as the plasma expands.

The average temperatures and densities inferred from the CRE analysis are shown in Figs. 2E and 2F, respectively. The analysis indicates that line emission is characterized by electron temperatures that remain low up until the peak of the power pulse, $(\sim 400 \mathrm{eV})$, while the ion density emitting the $\mathrm{K}$-shell radiation rises with the power pulse to a peak value of $\sim 3.5 \times 10^{20} \mathrm{~cm}^{-3}$. The low electron temperature inferred from the optically-thick line emission compared to the high temperature that is inferred from the optically-thin free-bound emission indicates that the hot plasma core, from which the free-bound emissions emanate (especially as they transit toward a cooler halo), is surrounded by a cooler plasma halo. In this halo, line emission from hotter, inner regions can be absorbed and reemitted, in which case the line emission reflects the lower average temperature of the halo. Note that line emission can still be copious from hot, nearly fully stripped intermediate plasma that is transitional between the core and the halo plasma. Recombination to excited states (electron onto bare nucleus) produces a free-bound continuum but is followed by cascade to the ground state producing characteristic line radiation. Also, the electron-collisional-excitation-rate coefficients from the hydrogen-like ground to excited states are greatly enhanced at high temperatures, allowing a fractional population of a few percent hydrogen-like ions to nonetheless produce detectable K-shell lines. These two excitational mechanisms are the source of the line radiation. 
After the peak of the power pulse when core expansion occurs, the ion density drops back to the values it had just before plasma assembly on axis, and the core and halo appear to merge as a consequence of the core expansion (Fig. 2B). During this period, the electron temperatures that are inferred from the CRE analysis are seen to rise rapidly to $\sim 1.2 \pm 0.2$ $\mathrm{keV}$, a value that is comparable to the peak temperature measured from the continuum slope. The drop in ion density during expansion implies a corresponding drop in plasma opacity. The inferred rise in electron temperature is thus correlated to a drop in opacity, which, in turn, would imply that line emissions from the hot core would more readily escape the plasma and be seen. The apparent merger of the two regimes spatially is consistent with the convergence of the spectrally determined line and continuum temperatures (compare Fig. 2D with Fig. 2B). This behavior appears to confirm that the core is now cooling faster than it can recombine. That is, in the hot but now less dense core, the ionization state of the plasma does not stay in equilibrium with the electrons due to long recombination times. Thus, the ionization state reflects the high electron temperatures that were present earlier at the time of peak compression. If plasmas the size of the radial halo widths are used in the above analysis, then the temperatures inferred up to peak power are $\sim 450 \mathrm{eV}$, while the peak density is reduced to $\sim 10^{20} \mathrm{~cm}^{-3}$. Similarly, the temperatures inferred after peak power rise by $100-200$ $\mathrm{eV}$ to $1.3-1.4 \mathrm{keV}$.

\section{Summary}

Although the results of the line analysis are somewhat flawed by the CRE assumption, the interpretation that can be drawn from the combined line plus-continuum analysis suggests that a lag in recombination following peak compression does occur. In particular, it suggests that the electrons cool faster than the ionization stages can recombine to remain in equilibrium. Thus, the line analysis itself supports the idea that its equilibrium assumption has broken down. In spite of some uncertainties in the size of the plasma to be used in the analysis and the axial variation not accounted for, the line analysis also supports the results of the continuum analysis. Namely, it produces a peak temperature that is comparable to that derived from the continuum-slope measurements. Furthermore, it implies the existence of subkilovolt temperatures in the plasma at a time when superkilovolt temperatures are present; that is, the combined analysis shows the existence of kilovolt temperature differences over millimeter distances that suggests the presence of perhaps magnetically-inhibited heat flow. In conclusion, this analysis shows the utility of the two diagnostics to infer electron temperatures from high-power z-pinch implosions. 


\section{References}

1. T. W. L. Sanford, T. J. Nash, B. M. Marder, R. Humphreys, C. Deeney, R. B. Spielman, J. F. Seamen, R. C. Mock, J. S. McGurn, D. Jobe, T. L. Gilliland, M. Vargas, K. Struve, W. A. Stygar, J. H. Hammer, J. L. Eddleman, J. S. De Groot, D. Mosher, K. G. Whitney, J. P. Apruzese, P. Pulsifer, Y. Maron, Bull. Am Phys. Soc. 40, 1846 (1995).

2. D. D. Bloomquist, R. M. Stinnett, D. H. McDaniel, J. R. Lee, A. W. Sharpe, J. A. Halbleib, L. G. Shlitt, P. W. Spence, and P. Corcoran, in Proceedings 6th IEEE Pulsed Power Conference Arlington, VA, edited by P. J. Truchi and B. H. Bernstein (IEEE, New York, 1987), p. 310.

3. J. H. Hammer, Bull. Am. Phys. Soc. 40, 1863 (1995) and Phys. Plasmas 3, 2063 (1996).

4. C. Deeney, P. D. LePell, B. H. Failor, S. L. Wong, J. P. Apruzese, K. G. Whitney, J. W. Thornhill, J. Davis, E. Yadlowsky, R. C. Hazelton, J. J. Moschella, T. Nash, and N. Loter, Phys. Rev. E 51(5), 4823 (1995).

5. R. B. Spielman, J. S. De Groot, T. J. Nash, J. McGurn, L. Ruggles, M. Vargas, and K. G. Estabrook, Dense z-Pinches (3rd International Conference, London, UK, 1993), AIP Conference Proceedings 299, editors H. Haines and A. Knight, American Institute of Physics, New York 1994, L.C. Catalog Card No. 93-74569, pages 404-420.

6. R. B. Spielman, Rev. Sci. Instrum. 66, 867 (1995).

7. R. B. Spielman, Sandia National Laboratories Technical Report SAND85-0699 (1990).

8. J. W. Thornhill, K. G. Whitney, C. Deeney, and P. D. Pell, Phys. Plasmas 1, 321 (1994).

9. M. C. Coulter, K. G. Whitney, and J. W. Thornhill, J. Quant. Spectrosc. Radiat. Transfer 44,443 (1990).

10. J. P. Apruzese, K. G. Whitney, J. Davis, and P. C. Kepple, J. Quant. Spectrosc. Radiat. Transfer (submitted 1996).

11. C. Deeney, T. Nash, R. R. Prasad, L. Warren, K. G. Whitney, J. W. Thornhill, and M. C. Coulter, Phys. Rev. A. 44, 6762 (1991); C. Deeney, P. D. LePell, I. Roth, T. Nash, L. Warren, R. R. Prasad, C. McDonald, F. K. Childers, P. Sincerny, M. C. Coulter, and K. G. Whitney, J. Appl. Phys. 72, 1297 (1992). 


\section{Distribution}

3 University of New Mexico

Dept. of Chemistry \& Nuclear Eng.

Attn: Prof. G. Cooper

Prof. S. Humphries

Prof. N. F. Roderick

Albuquerque, NM 87131

2 Ktech Corp.

Attn: T. Smith

T. Roemer

901 Pennsylvania NE

Albuquerque, NM 87110

6 Los Alamos National Laboratory

Attn: R. J. Macek

R. Stringfield

H. A. Thiessen

R. Fehl

D. L. Peterson

M. E. Jones

P. O. Box 1663

Los Alamos, NM 87545

1 W. J. Schafer Assoc., Inc.

Attn: D. C. Straw

2000 Randolph Road SE, Suite 205

Albuquerque, NM 87106

2 Mission Research Corporation

Attn: D. R. Welch

K. Struve

1720 Randolph Road SE

Albuquerque, NM 87106

1 North Star Research Corp.

Attn: R. J. Adler

5555 Zuni SE, Suite 345

Albuquerque, NM 87108
2 Titan Industries

Attn: R. B. Miller

J. Smith

P. O. Box 9254

Albuquerque, NM 87119

$5 \quad$ Fermilab

Attn: Prof. L. M. Lederman, Director Emeritus

J. H. Christenson

H. B. Jensen

T. E. Nash

M. Month

P. O. Box 500

Batavia, IL 60510

1 University of California Davis

Attn: Prof. John deGroot

Department of Applied Science

228 Walker Hall

Davis, CA 95616

7 Harry Diamond Laboratories

Aurora Facility

Attn: J. Corrigan, Director

M. Bushell

R. Fleetwood

K. G. Kerris

J. McGarrity

G. Merkel

M. Smith

2800 Powder Mill Road

Adelphi, MD 20783

1 Center for Radiation Research

Attn: S. M. Seltzer

National Bureau of Standards

Gaithersburg, MD 20899 


\section{Distribution (continued)}

23 Naval Research Laboratory

Attn: G. Cooperstein

D. Hinshelwood

D. Mosher

J. M. Neri

P. Ottinger

F. C. Young

R. Commisso

J. Davis

K. G. Whitney (10 copies)

J. P. Apruzese

P. E. Pulsifer

J. L. Giuliani

W. Thornhill

Washington, DC 20375

1 Stanford Linear Accelerator Center Attn: W. R. Nelson

P. O. Box 4349

Stanford, CA 94305

1 Air Force Institute of Technology Dept. of Engineering Physics

Attn: Prof. D. E. Beller

Wright-Patterson AFB, OH

45433-6583

1 Auburn University

Office of the Vice President for

Research

Attn: Prof. A. K. Hyder

Associate Vice President

202 Samford Hall

Auburn, AL 36849-5112

1 Columbia University

Physics Department

Attn: Prof. W. Lee

538 W. 120 Street

New York, NY 10027
2 Cornell University

Laboratory of Plasma Sciences

Attn: Prof. J. B. Greenly

Prof. D. A. Hammer

369 Upson Hall

Ithaca, NY 14853-7501

1 Massachusetts Institute of

Technology

Plasma Fusion Center

Attn: Prof. R. D. Petrasso

167 Albany St.

Cambridge, MA 01239

1 George Washington University

Department of Physics

Attn: Prof. B. L. Berman

211 Samson Hall

Washington, DC 20052

1 New York University

Physics Department

Attn: Prof. J. Sculli

4 Washington Place

New York, NY 10003

2 University of Maryland

Laboratory for Plasma and

Fusion Energy Studies

Attn: Prof. Moon-Jhong Rhee

Prof. M. Reiser

College Park, MD 20742

1 Imperial College

Blackett Laboratory

Attn: Prof. M. Haines

London SW7 2BZ

ENGLAND 


\section{Distribution (continued)}

2 Berkeley Research Associates

Attn: N. R. Pereira

$$
\text { J. Golden }
$$

P. O. Box 852

Springfield, VA 22150

1 Mission Research Corporation

Attn: V. A. J. van Lint

P. O. Drawer 719

Santa Barbara, CA 03102

6 Physics International Co.

Attn: H. Helava

J. Creedon

C. Gilman

Sik-Lam Wong

J. C. Riordan

P. Sincerny

2700 Merced Street

San Leandro, CA 94577

$4 \quad$ Pulse Science Inc.

Attn: V. Bailey

I. Smith

J. Fockler

P. Spence

$600 \mathrm{McC}$ ormick St.

San Leandro, CA 94577

3 Science Applications, Inc.

Attn: A. A. Mondelli

D. Bacon

A. Manofsky

1710 Goodridge Drive

P. O. Box 1303

McLean, VA 22101

12 Lawrence Livermore National

Laboratory

Attn: P. T.Springer
A. Toor
S. Maxon
M. D. Rosen

M. Tabak

J. H. Hammer

J. L. Eddleman

G. B. Zimmerman

R. S. Thoe

J. Emig

C. Bruns

J. Hernandez

P. O. Box 808

Livermore, CA 94550

6 Atomic Weapons Research

Establishment

Attn: M. Goodman

N. Fenner

M. Gunerson

B. Harris

J. C. Martin

G. Wilson

Aldermaston, Reading RG7 4PR

Berkshire

ENGLAND

1 Culham Laboratory

Culham Lightning Studies Unit

Attn: C. J. Hardwick

Abingdon, Oxfordshire OX14 3DB

ENGLAND

3 Rutherford Appleton Laboratory

Attn: T. G. Walker, Director

T. Broome

C. Damerell

Chilton, Didcot

Oxon. OX11 OQx

ENGLAND

1 University des Sciences et

Techniques du Languedoc

Centre d'Elecronique de Montpellier

Attn: Prof. J. Gasiot

Place E. Battaillon

F-34 060 Montpellier Cedex

FRANCE 
Distribution (continued)

1 Weizmann Institute

1 MS 1165

Department of Nuclear Physics

Attn: Y. Maron

Rehovot 76100 Israel

1 MS 1170

P. S. Raglin, 9304

1 MS 1178

P. Nelson, 9305

1 MS 1159

J. J. Ramirez, 9310

1 MS 1159

M. A. Hedemann, 9311

4 Kernforshungszentrum Karlsruhe

1 MS 1160

G. J. Lockwood, 9311

Gmbh.

Attn: W. Bauer

1 MS 1160

K. Gilbert, 9312

1 MS 1153

H. Bluhm

P. Hoppe

H. U. Karow

1 MS 1179

J. W. Schwarz, 9312

M. T. Buttram, 9323

1 MS 1179

W. P. Ballard, 9341

1 MS 1179

D. E. Beutler, 9341

Postfach 3640, D-7500 Karlsruhe 1

1 MS 1179

R. S. Klingler, 9341

FEDERAL REPUBLIC OF

GERMANY

1 MS 1179

1 MS 1179

J. A. Halbleib, 9341

1 MS 1179

R. P. Kensek, 9341

J. R. Lee, 9341

6

C. E. R. N.

CH-1211

Attn: W. Blum

V. Chabaud

G. Jarlskog

G. Lutz

W. Manner

P. Weilhammer

Geneva 23

1 MS 1179

L. J. Lorence, 9341

1 MS 1106

T. F. Wrobel, 9341

1 MS 1106

A. W. Sharpe, 9342

1 MS 1106

R. Pepping, 9342

MS 1106

B. Peyton, 9342

P. Reilly, 9342

1 MS 1106

1 MS 1106

R. Broyles, 9342

1 MS 1106

B. Henderson, 9342

1 MS 1166

R. E. Craven, 9342

SWITZERLAND

$\begin{array}{lll}1 & \text { MS } & 0321 \\ 1 & \text { MS } & 1188 \\ 1 & \text { MS } & 0952 \\ 1 & \text { MS } & 0463 \\ 1 & \text { MS } & 0479 \\ 1 & \text { MS } & 0457 \\ 1 & \text { MS } & 0458 \\ 1 & \text { MS } & 1143 \\ 1 & \text { MS } & 1141 \\ 1 & \text { MS } & 1145 \\ 1 & \text { MS } & 1145 \\ 1 & \text { MS } & 1183 \\ 1 & \text { MS } & 0151 \\ 1 & \text { MS } & 1165 \\ 1 & \text { MS } & 1158 \\ 1 & M S & 1170 \\ 1 & \text { MS } & 1155\end{array}$

E. H. Barsis; 1400

F. McNamara, 1815

D. J. Miller, 2161

R. L. Hagengruber, 5000

L. M. Choate, 5151

R. J. Eagan, 5600

J. S. Rottler, 5607

J. K. Rice, 6500

T. R. Schmidt, 6502

R. J. Lipinski, 6514

R. L. Coats, 6514

K. W. Hanks, 6911

G. Yonas, 9000

J. E. Powell, 9300

J. H. Renken, 9301

G. A. Zawadzkas, 9302

W. Beezhold, 9303
1 MS 1190

1 MS 1195

1 MS 1178

1 MS 1178

1 MS 1178

1 MS 1182

1 MS 1193

1 MS 1193

1 MS 1193

1 MS 1193

1 MS 1193

1 MS 1193

1 MS 1193

1 MS 1193

1 MS 1193

1 MS 1193

1 MS 1193

16 MS 1193

1 MS 1187
G. J. Scrivner, 9352

D. L. Cook, 9500

J. P. Quintenz, 9502

R. Hamil, 9512

J. P. Corley, 9512

S. Drennan, 9512

B. N. Turman, 9521

J. E. Maenchen, 9531

M. T. Crawford, 9531

M. E. Cuneo, 9531

W. E. Fowler, 9531

D. L. Hanson, 9531

D. J. Johnson, 9531

M. G. Mazarakis, 9531

P. R. Menge, 9531

R. C. Mock, 9531

T. J. Renk, 9531

D. C. Rovang, 9531

T. W. L. Sanford, 9531

T. A. Mehlhorn, 9533 
Distribution (continued)

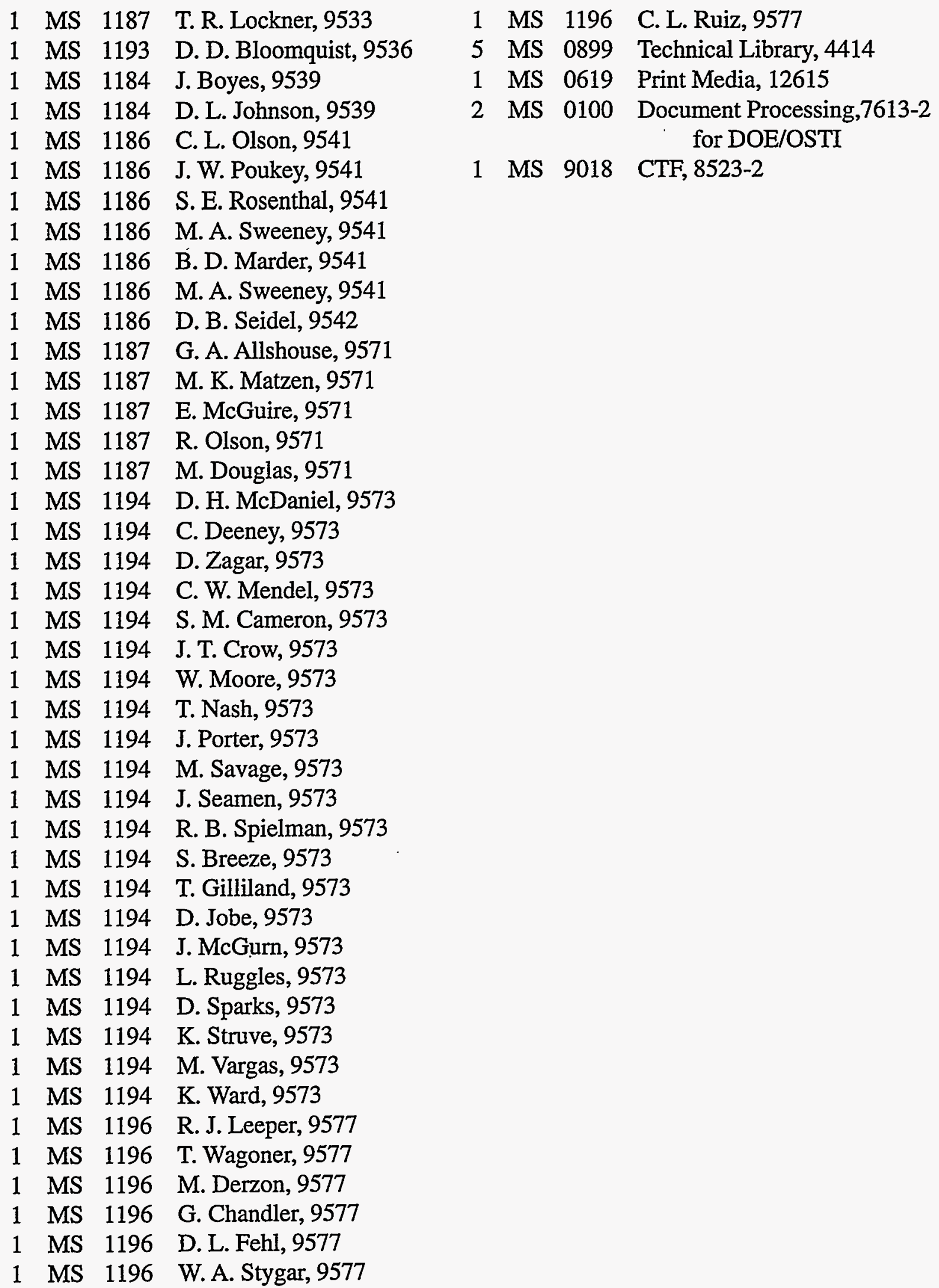

